\title{
Erratum to: The role of particle size of glyburide crystals in improving its oral absorption
}

Wenqian Yang ${ }^{1} \cdot Y_{0}$ gjun Wang ${ }^{1} \cdot$ Qiang Fu $^{1} \cdot$ Zhibin Guo $^{1} \cdot$ Bingjun Sun $^{1} \cdot$ Wen Liu $^{1}$. Yaxuan Liu ${ }^{1} \cdot$ Simeng Mu$^{1} \cdot$ Mengran Guo ${ }^{1} \cdot$ Jingru Li $^{1} \cdot$ Xiaohui Pu ${ }^{1} \cdot$ Zhonggui He $^{1}$

Published online: 1 May 2017

(C) Controlled Release Society 2017

Erratum to: Drug Deliv. and Transl. Res. (2017) 7:428-438

DOI 10.1007/s13346-017-0378-3

In the original article the order of the first and second authors is incorrect. It is corrected as shown in this erratum.

The online version of the original article can be found at http://dx.doi: 10 . 1007/s13346-017-0378-3

Yongjun Wang

i_maple@163.com

1 Shenyang Pharmaceutical University, Shenyang, People's Republic of China 